\title{
Eficacia y seguridad de dos esquemas de tratamiento de isotretinoína en adultos con rosácea: ensayo clínico aleatorizado
}

\author{
Efficacy and safety of two isotretinoin \\ treatment schemes in adults with \\ rosacea: randomized clinical trial
}

\author{
Fermín Jurado-Santa Cruz, ${ }^{\star}$ Tania Raisha Torres-Victoria, ${ }^{*}$ María Luisa Peralta-Pedrero, ${ }^{*}$ \\ María Julia Garza-Zúñiga, ${ }^{*}$ Martha Alejandra Morales-Sánchez ${ }^{*}$
}

\section{RESUMEN}

Introducción: La rosácea es una enfermedad inflamatoria cutánea que afecta la calidad de vida. La isotretinoína es una alternativa terapéutica en casos graves. Existe controversia sobre la eficacia de dosis bajas de isotretinoína para el tratamiento de la rosácea pápulo-pustular. Material y métodos: Ensayo clínico aleatorizado que reclutó 68 pacientes con rosácea pápulo-pustular. Los participantes se asignaron aleatoriamente a dos dosis de isotretinoína, el grupo A recibió $20 \mathrm{mg} /$ día y el grupo $B, 10$ $\mathrm{mg} /$ día durante 12 semanas. Las variables de respuesta fueron el conteo de lesiones, el nivel de eritema y melanina a las cero, cuatro, ocho y 12 semanas. Se midió el cambio en la calidad de vida a las 12 semanas y los efectos adversos durante todo el estudio. La recidiva se evaluó a las 24 semanas. Resultados: La reducción en el número de lesiones fue similar en ambos grupos, al igual que la incidencia de efectos adversos. La calidad de vida mejoró en ambos grupos al igual que el nivel de eritema. El nivel de melanina fue menor al final del tratamiento en el grupo que recibió $20 \mathrm{mg} / \mathrm{día}$ de isotretinoína. Conclusiones: La eficacia y seguridad de ambos esquemas de dosis de isotretinoína es similar, por lo que se recomienda el empleo de $10 \mathrm{mg} /$ día de isotretinoína para pacientes con rosácea pápulo-pustular.

Palabras clave: Rosácea, isotretinoína, calidad de vida, ensayo clínico.

\section{ABSTRACT}

Introduction: Rosacea is an inflammatory skin disease that affects quality of life. Isotretinoin is a therapeutic alternative in severe cases. Controversy exists about the efficacy of low-dose isotretinoin for the treatment of papulo-pustular rosacea. Material and methods: Randomized clinical trial enrolling 68 patients with papulo-pustular rosacea. Participants were randomly assigned to two doses of isotretinoin, group A received $20 \mathrm{mg} /$ day and group $B 10 \mathrm{mg} /$ day for 12 weeks. The response variables were the lesion count, the level of erythema and melanin at zero, four, eight and 12 weeks. Change in quality of life at 12 weeks and adverse effects were measured throughout the study. Recurrence was assessed at 24 weeks. Results: The reduction in the number of lesions were similar in both groups, as well as the incidence of adverse effects. The quality of life improved in both groups, as did the level of erythema. The level of melanin was lower at the end of the treatment in the group that received $20 \mathrm{mg} /$ day of isotretinoin. Conclusions: The efficacy and safety of both isotretinoin dose schedules are similar, due to this results the use of isotretinoin $10 \mathrm{mg} /$ day is recommended for patients with papulo-pustular rosacea.

Keywords: Rosacea, isotretinoin, quality of life, clinical trial.
* Unidad de Enseñanza e Investigación, Centro Dermatológico «Dr. Ladislao de la Pascua", Servicios de Salud Pública de la Ciudad de México, Ciudad de México, México.
Citar como: Jurado-Santa Cruz F, Torres-Victoria TR, Peralta-Pedrero ML, GarzaZúñiga MJ, Morales-Sánchez MA. Eficacia y seguridad de dos esquemas de tratamiento de isotretinoína en adultos con rosácea: ensayo clínico aleatorizado. Rev Cent Dermatol Pascua. 2020; 29 (2-3): 47-55. https://dx.doi.org/10.35366/99916 


\section{INTRODUCCIÓN}

La rosácea es una enfermedad inflamatoria cutánea caracterizada por eritema, telangiectasias, pápulas y pústulas en cara; de predominio en frente, mejillas, nariz y mentón. Puede ocasionar cambios fimatosos secundarios a hipertrofia de las glándulas sebáceas y fibrosis e inflamación ocular. ${ }^{1,2}$ Predomina entre la segunda y tercera décadas de la vida; afecta más a mujeres que a hombres. ${ }^{3}$ Una revisión sistemática en el 2018 calculó la prevalencia global de rosácea en $5.46 \%$ en la población adulta. ${ }^{4}$ Es más prevalente en adultos con fototipos claros (Fitzpatrick I y II). ${ }^{5}$ En fototipos oscuros, la rosácea puede estar subestimada, ya que el eritema y las telangiectasias son difíciles de detectar, la prevalencia de rosácea en raza negra es de $2 \%$ y en población latina de $3.9 \%{ }^{6}$

En la rosácea se ha demostrado que existe una falla en la regulación del sistema inmune innato que provoca una inflamación excesiva y vasodilatación en respuesta a factores ambientales. ${ }^{7}$ El diagnóstico se sustenta en la exploración física; de acuerdo a las lesiones que predominan se clasifica en: eritemato-telangiectásica, pápulo-pustular, fimatosa y ocular. El criterio diagnóstico más importante de acuerdo con la Sociedad Nacional de Rosácea es el eritema centro-facial fijo, que puede intensificarse periódicamente. Las manifestaciones secundarias reconocidas son la sensación urente o de adormecimiento, edema y xerosis centro-facial. ${ }^{8}$ Como enfermedad cutánea, la rosácea impacta de forma negativa en la calidad de vida del paciente en la esfera emocional y social, así como la autopercepción y autoestima. ${ }^{9}$

El tratamiento actual de la rosácea está enfocado en controlar las manifestaciones clínicas con medicamentos tópicos, orales y/o dispositivos de luz; aunado al cuidado de la piel y cambios en el estilo de vida. ${ }^{10}$ Las guías de práctica clínica recomiendan el uso de doxiciclina a dosis bajas y metronidazol tópico o ácido azelaico, como la primera línea de tratamiento en la rosácea pápulo-pustular moderada a grave. Sin embargo, se reportan recaídas posteriores a la interrupción de la doxiciclina. Otros tratamientos eficaces son: ivermectina tópica, brimonidina tópica, tetraciclinas vía oral, isotretinoína, láser y luz pulsada. ${ }^{2}$ Estudios previos han demostrado la efectividad de la isotretinoína para la rosácea pápulo-pustular grave o resistente a tratamiento con tetraciclinas. Las dosis empleadas en la mayoría de los ensayos clínicos han sido similares a las administradas en pacientes con acné $(0.5-1 \mathrm{mg} /$ $\mathrm{kg} / \mathrm{día}$ ). No obstante, estudios más recientes han de- mostrado la efectividad de la isotretinoína a dosis bajas (0.1-0.3 $\mathrm{mg} / \mathrm{kg} / \mathrm{día})$ para el tratamiento de la rosácea pápulo-pustular, con la ventaja de la disminución de la incidencia de los efectos adversos. ${ }^{11-13}$. Ante esta evidencia científica, el objetivo de este estudio fue evaluar la eficacia de la isotretinoína a dosis de $10 \mathrm{mg} /$ día, en comparación con la dosis habitual de $20 \mathrm{mg} /$ día para el tratamiento de la rosácea pápulo-pustular moderada a grave en adultos.

\section{MATERIAL Y MÉTODOS}

Se realizó un ensayo clínico aleatorizado en adultos (18 a 59 años de edad) con rosácea pápulo-pustular moderada a grave del Centro Dermatológico «Dr. Ladislao de la Pascua». El estudio fue aprobado por los Comités de Investigación y Ética en Investigación institucionales. Todos los pacientes aceptaron participar voluntariamente y firmaron carta de consentimiento informado. Se consideró que un paciente presentaba rosácea pápulo-pustular moderada a grave cuando en la evaluación global obtenía dos o tres puntos en eritema, pápulas, pústulas y telangiectasias. Los criterios de exclusión fueron los siguientes: tratamiento durante los últimos tres meses (orales, tópicos y/o láser), tabaquismo, consumo frecuente (semanal) de bebidas alcohólicas, formas especiales de rosácea como extrafacial y pioderma facial, pacientes con enfermedades contraindicadas o que pueden empeorar con el uso de isotretinoína, tales como insuficiencia renal, hepática, hipervitaminosis A o dislipidemia. Se tomaron estudios de laboratorio (biometría hemática, prueba de función hepática, perfil de lípidos, CPK y hormona gonadotropina coriónica humana fracción beta en mujeres) antes del reclutamiento para evaluar su elegibilidad. Todas las mujeres fueron enviadas al ginecólogo para el inicio de un método anticonceptivo.

Los participantes que cumplieron los criterios de selección del estudio fueron asignados aleatoriamente (lista creada en sealedenvelope.com) a alguno de los dos grupos de intervención: el grupo A recibió 20 $\mathrm{mg}$ de isotretinoína al día, mientras que el grupo $B$ recibió $10 \mathrm{mg}$ al día, durante 12 semanas. Los participantes fueron evaluados a las cero, cuatro, ocho y 12 semanas de la intervención por un médico dermatólogo encargado de identificar y realizar el conteo de lesiones faciales. La variable de resultado principal fue el cambio en el número de lesiones inflamatorias y no inflamatorias. El eritema persistente y las telangiectasias se calificaron en una escala del 0 al 3 de acuerdo a su intensidad y frecuencia. También se 
cuantificó el índice de eritema y melanina (escala del 0 al 999) con la sonda Mexameter MX18 (Courage + Khazaka electronic $\mathrm{GmbH}$ ). El mexámetro es un equipo que mide los dos principales componentes que dan el color a la piel: hemoglobina y melanina, a través de los principios de absorción y reflexión. Este equipo emite tres longitudes de onda de luz específicas y un receptor mide la luz reflejada. Al definirse la cantidad de luz emitida es posible calcular la luz absorbida por la piel. Se evaluó la calidad de vida utilizando la versión en español del cuestionario Dermatology Life Quality Index (DLQI), que a mayor puntaje indica una menor calidad de vida. Dicho cuestionario es un instrumento que consta de 10 ítems, cuyo marco temporal abarca los últimos siete días. Cada una de las preguntas incluye cuatro posibles respuestas con puntuaciones del 0 al 3. Las dimensiones de la calidad de vida que mide son: síntomas y percepciones, actividades diarias, ocio, trabajo/estudio, relaciones interpersonales y tratamiento. La puntuación va desde cero, que se traduce en un impacto mínimo en la calidad de vida, hasta 30 puntos, que refleja el máximo impacto en la calidad de vida. El conteo de lesiones, la medición de eritema, melanina, los estudios de laboratorio y la toma de fotografías se realizó a las cero, cuatro, ocho y 12 semanas del estudio. La calidad de vida se midió a las cero y 12 semanas, mientras que la recidiva se evaluó a las 24 semanas. Se definió recidiva como la presencia de más lesiones que las reportadas a las 12 semanas. Los efectos adversos se midieron por medio de evaluaciones clínicas y mediante bitácoras, donde se registró el día de inicio del síntoma y mediante pruebas de laboratorio durante las visitas cuatro, ocho y 12 . El criterio para interrumpir las intervenciones fueron las alteraciones grado 2 de los resultados de los estudios de laboratorio, establecidos por el Instituto Nacional de Cáncer 2003, definidos como grado 2 aquéllos con los siguientes valores: triglicéridos $376-750 \mathrm{mg} / \mathrm{dL}$, colesterol total $301-400 \mathrm{mg} / \mathrm{dL}$, ALT/AST $101-200 \mathrm{mg} /$ $\mathrm{dL}$, leucocitos 2.0 a $2.9 \times 10^{3} / \mathrm{mL}$, plaquetas $50-74 \times$ $10^{3} / \mathrm{mL}$ y hemoglobina $8-9.9 \mathrm{~g} / \mathrm{dL}$.

El tamaño de muestra se calculó utilizando la fórmula para comparar dos proporciones, considerando una diferencia en el número de lesiones inflamatorias entre ambos grupos de $10 \%$, alfa de 0.5 y beta de $20 \%$, se consideraron pérdidas durante el seguimiento de $20 \%$. Los datos fueron analizados con el programa SPSS versión 21.0 (SPSS Chicago, II). Las variables cualitativas se describieron mediante frecuencias y las cuantitativas con distribución normal utilizando la media y la desviación estándar; en caso contrario, se calculó la mediana y el rango intercuartílico (RIQ). Para analizar las diferencias en el conteo de lesiones, índices de eritema, melanina y calidad de vida se utilizó la prueba t de Student en caso de distribución normal, o la U de Mann-Whitney en caso contrario. Se consideró que existían diferencias estadísticamente significativas (entre los grupos) con un resultado de alfa menor a 0.05.

\section{RESULTADOS}

Se reclutaron 34 pacientes por grupo (Figura 1). En el grupo A, donde se otorgó $20 \mathrm{mg} /$ día de isotretinoína, $70.5 \%$ completaron el seguimiento a 12 semanas. Hubo 10 pérdidas, seis pacientes no acudieron a las citas de seguimiento (semana ocho y/o 12), dos pacientes suspendieron por decisión propia, un paciente refirió mialgias sin elevación de CPK y un paciente tuvo elevación de CPK junto con mialgias, por lo que se decidió su suspensión. En el grupo B, donde recibieron $10 \mathrm{mg} /$ día de isotretinoína, $73.5 \%$ completaron el tratamiento, hubo nueve pérdidas de seguimiento. De éstos, siete pacientes no acudieron a las citas de control (semana ocho y/o 12), un paciente refirió síntomas depresivos, por lo que se decidió su suspensión y un paciente tuvo elevación en el valor de bilirrubinas (BT $2.7 \mathrm{mg} / \mathrm{dL}$ ).

No hubo diferencias estadísticamente significativas en cuanto a las características basales en ambos grupos de tratamiento (Tabla 1). Predominó el sexo femenino en ambos grupos, con más de $60 \%$ de los casos.

\section{Eficacia}

Al comparar los resultados en ambos grupos a la semana 12 de seguimiento, se encontró un mayor número de pápulas y pústulas en el grupo A (isotretinoína $20 \mathrm{mg}$ ) con una mediana de 29 pápulas (RIQ 20-41), y de 22 pápulas (RIQ 15-38) en el grupo $B$ (isotretinoína $10 \mathrm{mg}$ ), $p=0.248$. La mediana de pústulas contabilizadas fue de siete en el grupo A (RIQ 2-15) y de cinco en el grupo $B$ (RIQ2-10), $p=0.519$. La mediana de la calificación de las telangiectasias basales en el grupo $A$ fue de uno y en el grupo $B$ de dos, mientras que a la semana 12 fue de uno en ambos grupos, sin significancia estadística. El eritema transitorio basal en ambos grupos fue similar, dos (rango 2-3) y a las 12 semanas fue el mismo para ambos, dos (1-2), $p=0.694$. Al comparar los resultados entre las visitas cuatro, ocho y 12 (Figuras 2 y 3), no se encontraron diferencias significativas en cuanto a pápulas, pústulas, telangiectasias o eritema transitorio entre los grupos de intervención (Tabla 2, Figuras 4 y 5). 

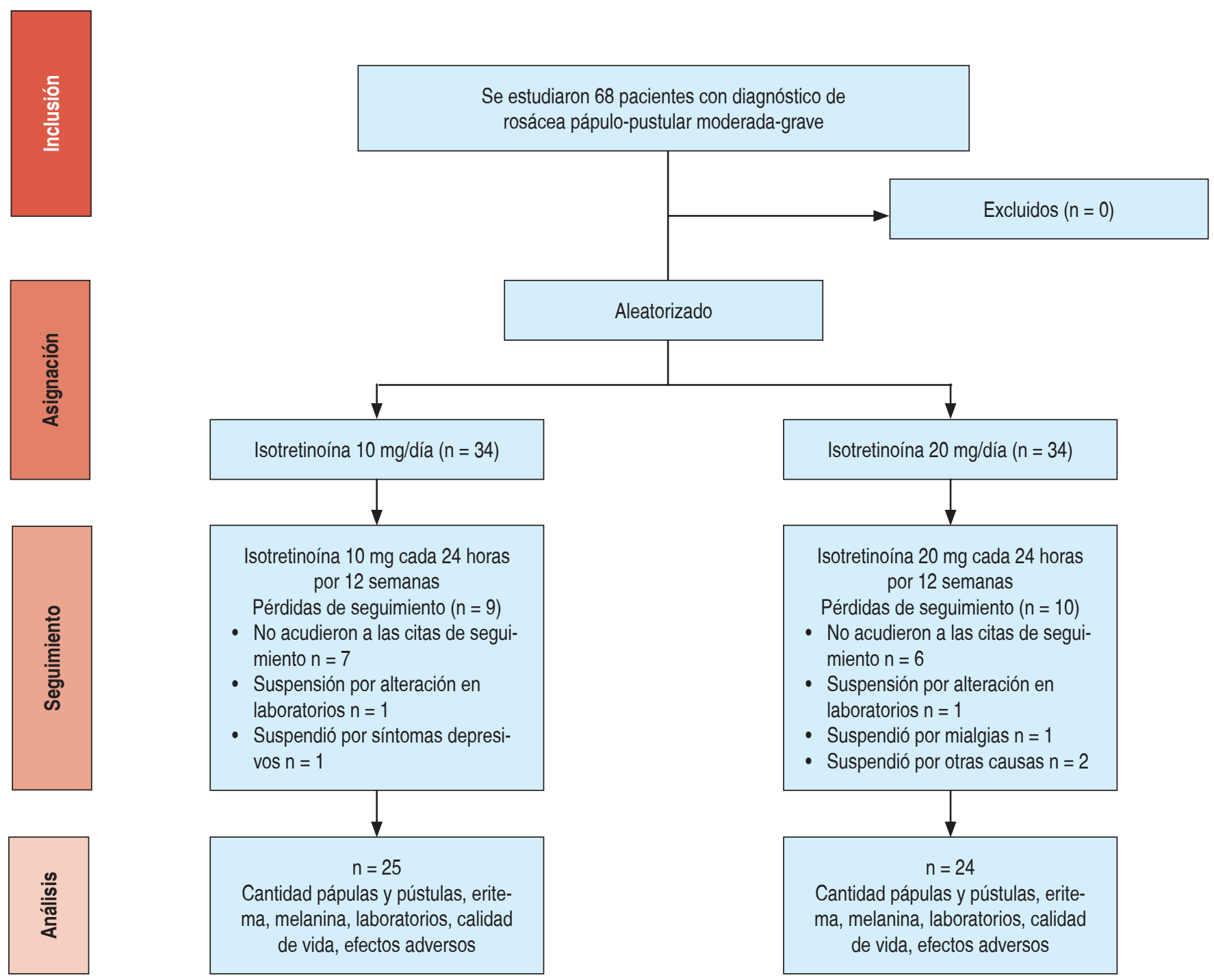

Figura 1: Diagrama de flujo del estudio de acuerdo a CONSORT.

Tabla 1: Comparación entre grupos.

\begin{tabular}{lccc} 
& $\begin{array}{c}\text { Grupo A } \\
(20 \mathrm{mg} / \mathrm{día})\end{array}$ & $\begin{array}{c}\text { Grupo B } \\
(10 \mathrm{mg} / \mathrm{día})\end{array}$ & $\mathrm{p}$ \\
\hline Edad (años) & $36 \pm 10$ & $39 \pm 10$ & 0.197 \\
Sexo femenino (\%) & 81.8 & 66.7 & 0.130 \\
Pústulas basales $^{*}$ & $7(2-15)$ & $5(2-10)$ & 0.519 \\
Pápulas basales $^{*}$ & $29(20-41)$ & $22(15-38)$ & 0.248 \\
DLQl $^{*}$ & $6.5(3.0-11.0)$ & $7.0(3.5-11.5)$ & \\
\hline
\end{tabular}

*Mediana y rango intercuartílico.

DLQI = Dermatology Life Quality Index.
En cuanto a los resultados de los estudios de laboratorio, sólo se encontró diferencia estadísticamente significativa en el valor de colesterol total, triglicéridos, GGT y LDL-C (Tabla 3), pero sin relevancia clínica. Se observó una elevación significativa del colesterol total a la semana ocho en el grupo A ( $20 \mathrm{mg} / \mathrm{día})$, con un colesterol total de $179 \pm 33 \mathrm{mg} / \mathrm{dL}$; mientras que el grupo B tuvo valores de $157 \pm 30 \mathrm{mg} / \mathrm{dL},(p<0.05)$. A la semana cuatro hubo una elevación significativa en el grupo A de los niveles de triglicéridos, con una mediana de $133 \mathrm{mg} / \mathrm{dL}$ (RIQ 99-183); por su parte, en el grupo B fue de $100 \mathrm{mg} / \mathrm{dL}$ (RIQ 76-130). EI LDL-C estuvo elevado a la semana ocho con un valor promedio de $121 \pm 32$ en el grupo $A$ y en el grupo $B$ de 98 $\pm 35(p<0.05)$. A la semana cuatro, la mediana del valor 
Pápulas

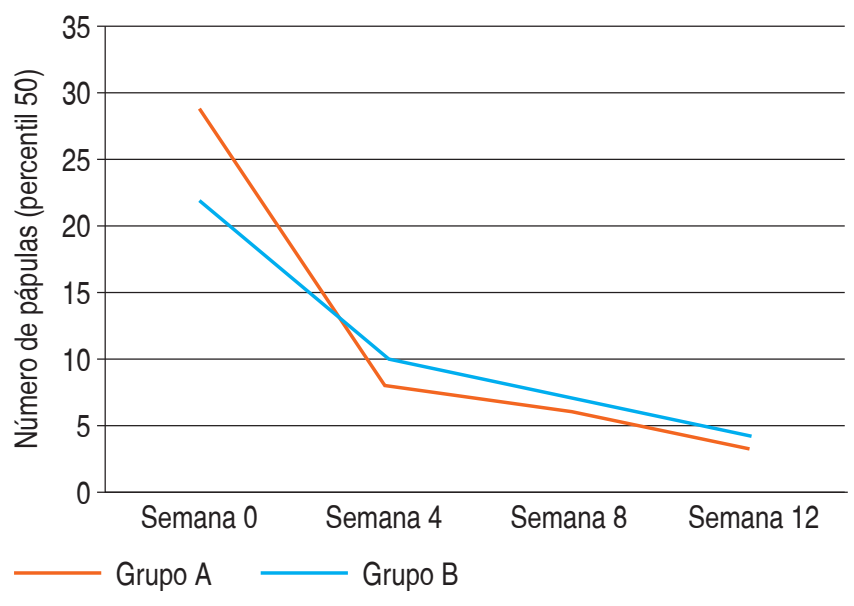

Figura 2: Número de pápulas por visita.

Pústulas

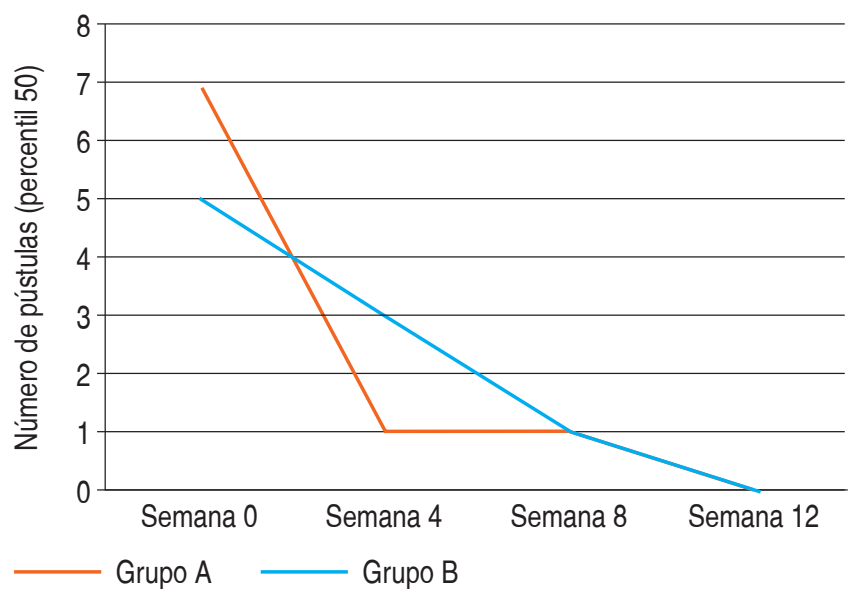

Figura 3: Número de pústulas por visita.

de gamma-glutamil transferasa (GGT) en el grupo $A$ fue de $27 \mathrm{mg} / \mathrm{dL}$ (RIQ 20 a 40 ) y el grupo B de $21 \mathrm{mg} / \mathrm{dL}$ (RIQ 13-29). Aunque hubo diferencias estadísticamente significativas de algunos parámetros de laboratorio, con valores más altos en el grupo $A$, este aumento de los niveles no fue clínicamente significativo ni ameritó la interrupción del tratamiento en ninguno de los dos grupos.

Se encontraron diferencias en el valor de melanina a las semanas ocho y 12 . El grupo A ( $20 \mathrm{mg} /$ día) tuvo una mediana de 198 (RIQ 173-246); mientras que el B (10 mg/día) tuvo una mediana de 213 (RIQ 194-246), p $<0.05$. En la semana 12 el grupo A tuvo una mediana de 176 (RIQ 141-212); mientras que la del grupo $B$ fue de 208 (RIQ 180-241), p < 0.05 (Tabla 4).

\section{Efectos adversos}

Los efectos adversos se valoraron a la semana cuatro, ocho y 12 (Tabla 5). A la semana cuatro, $58.8 \%$ de los pacientes en el grupo B con $10 \mathrm{mg} /$ día presentaron algún síntoma. El principal síntoma fue la xerosis en $29.4 \%$ de los casos. En el grupo A (20 mg/día), 52.9\% presentó algún efecto adverso sin observarse diferencias estadísticamente significativas entre los grupos. En este grupo, los síntomas principales fueron la xerosis, y el dolor en $23.5 \%$ cada uno, y prurito en $14.7 \%$. A la semana ocho, los efectos adversos presentados fueron menores, siendo $26.5 \%$ en ambos grupos y predominando la xerosis con $8.8 \%$ en el grupo de $20 \mathrm{mg} / \mathrm{día}$ de isotretinoína y en $14.7 \%$ del grupo de isotretinoína $10 \mathrm{mg} /$ día. A la semana 12, 5.9\% de los pacientes en ambos grupos presentaron algún efecto adverso. No se presentaron diferencias ni clínicas ni estadísticamente significativas entre ambos grupos.

\section{Calidad de vida}

La calidad de vida se midió en la visita basal y en la semana 12, al término del tratamiento. No se documentaron diferencias en los puntajes de la calidad de vida entre los grupos de intervención. Se compararon las preguntas

\begin{tabular}{|c|c|c|c|c|}
\hline Variables & Basal & Semana 4 & Semana 8 & Semana 12 \\
\hline \multicolumn{5}{|l|}{ Pápulas (n) } \\
\hline Grupo A & $29(20-41)$ & $8(3-18)$ & $6(3-14)$ & $3(2-8)$ \\
\hline Grupo B & $22(15-38)$ & $10(8-17)$ & $7(4-16)$ & $4(1-10)$ \\
\hline \multicolumn{5}{|l|}{ Pústulas (n) } \\
\hline Grupo A & $7(2-15)$ & $1(0-4)$ & $1(0-3)$ & $0(0-1)$ \\
\hline Grupo B & $5(2-10)$ & $3(0-5)$ & $1(0-4)$ & $0(0-2)$ \\
\hline \multicolumn{5}{|c|}{ Telangiectasias $(\mathrm{n})$} \\
\hline Grupo A & $1(1-2)$ & $1(1-2)$ & $1(1-2)$ & $1(1-2)$ \\
\hline Grupo B & $2(1-2)$ & $2(1-2)$ & $2(1-2)$ & $1(1-2)$ \\
\hline \multicolumn{5}{|c|}{ Eritema transitorio $(\mathrm{n})$} \\
\hline Grupo A & $2(2-3)$ & $2(2-3)$ & $2(2-3)$ & $2(1-2)$ \\
\hline Grupo B & $2(2-3)$ & $2(1-2)$ & $2(1-3)$ & $2(1-2)$ \\
\hline \multicolumn{5}{|c|}{ Eritema no transitorio (n) } \\
\hline Grupo A & $2(2-3)$ & $2(2-3)$ & $2(2-3)$ & $2(1-2)$ \\
\hline Grupo B & $3(2-3)$ & $2(2-2)$ & $2(1-3)$ & $2(2-2)$ \\
\hline \multicolumn{5}{|l|}{ DLQI (n) } \\
\hline Grupo A & $6.5(3.0-11.0)$ & - & - & $1.0(0.0-3.0)$ \\
\hline Grupo B & $7.0(3.5-11.5)$ & - & - & $1.0(0.0-3.0)$ \\
\hline
\end{tabular}



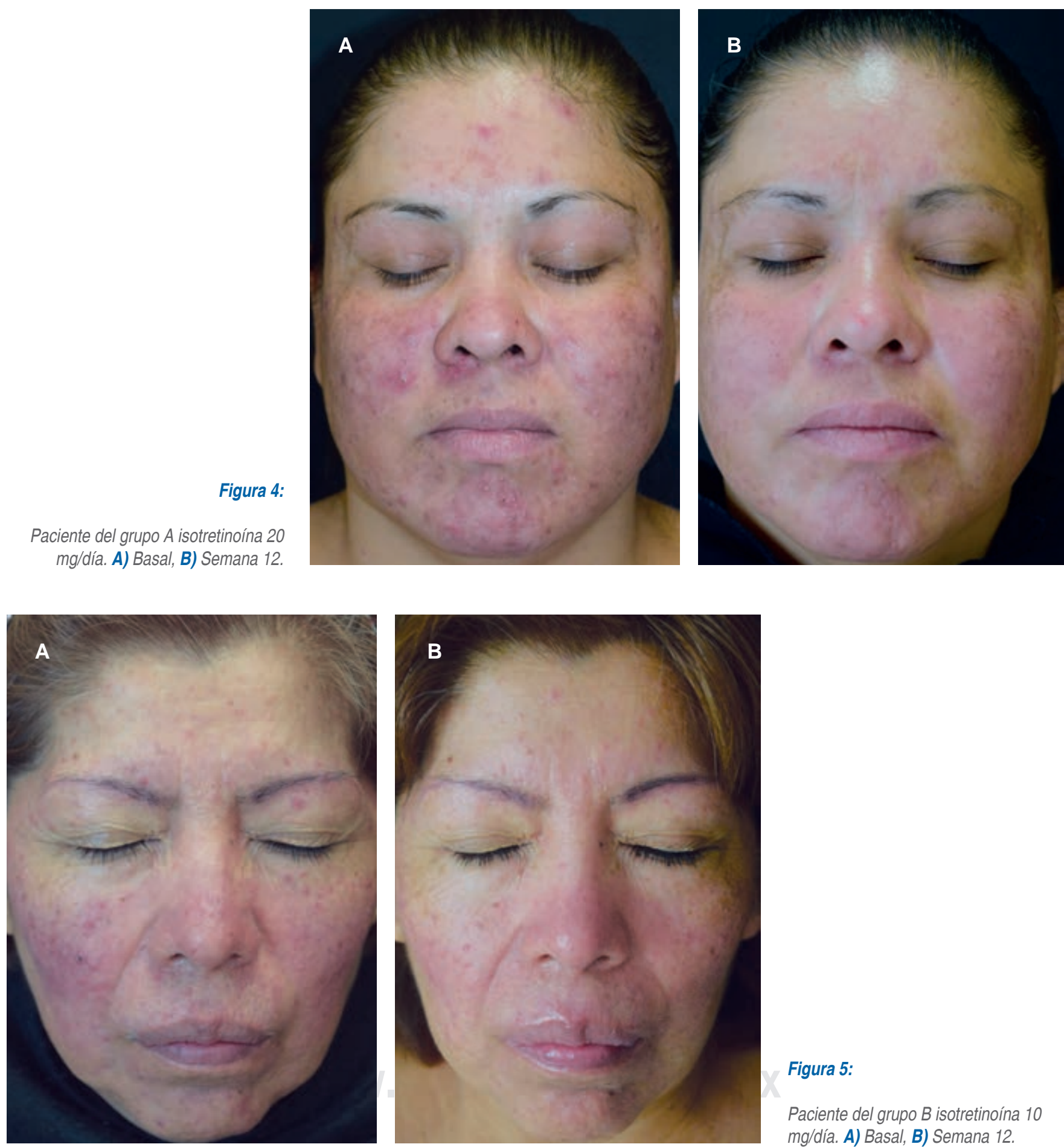

Figura 5:

Paciente del grupo B isotretinoína 10 mg/día. A) Basal, B) Semana 12.

entre ambos grupos de manera individual de las semanas cero y 12 , y no se encontraron diferencias. En la primera visita en el grupo A, la mediana del puntaje fue de 6.5 (RIQ 3.0-11.0) mientras que la del grupo B fue de 7 (RIQ
3.5-11.5). Aunque no se encontraron diferencias estadísticamente significativas sí hubo una disminución en los puntajes de la calidad de vida a las 12 semanas en ambos grupos, con una disminución promedio de un punto (0-3). 


\section{Recidiva a la semana 24}

Se realizó seguimiento a los pacientes que acudieron a las 12 semanas posteriores al término de las intervenciones. Se definió recaída a la semana 24 como la presencia de un mayor número de lesiones inflamatorias (pápulas o pústulas) en comparación con la semana 12. Del grupo B (10 mg/día) acudieron 16/25 (64\%), mientras que del grupo A (20 mg/día) acudieron 7/24
(29\%). En el grupo B, los pacientes que recibieron la dosis más baja (10 mg/día), 68\% (11/16) presentaron recaída, mientras que en el grupo A (20 mg/día) $71 \%$ la presentó.

\section{DISCUSIÓN}

La relevancia de este estudio radica en que los resultados obtenidos arrojan que no hay una diferencia signifi-

\begin{tabular}{|c|c|c|c|c|}
\hline Variables & Basal & Semana 4 & Semana 8 & Semana 12 \\
\hline \multicolumn{5}{|l|}{ AST (U/L) } \\
\hline Grupo A & $20(15-25)$ & $23(14-44)$ & $22(13-28)$ & $20(18-23)$ \\
\hline Grupo B & $20(15-34)$ & $20(16-27)$ & $20(13-32)$ & $20(17-25)$ \\
\hline \multicolumn{5}{|l|}{ ALT (U/L) } \\
\hline Grupo A & $20(17-28)$ & $22(17-29)$ & $19(17-25)$ & $18(13-24)$ \\
\hline Grupo B & $19(17-26)$ & $21(16-25)$ & $19(16-25)$ & $21(14-28)$ \\
\hline \multicolumn{5}{|l|}{ GGT (U/L) } \\
\hline Grupo A & $24(15-44)$ & $27(20-40)$ & $26(18-40)$ & $24(17-37)$ \\
\hline Grupo B & $19(13-26)$ & $21(13-29)$ & $20(14-34)$ & $21(14-30)$ \\
\hline \multicolumn{5}{|c|}{ Bilirrubina total (mg/dL) } \\
\hline Grupo A & $0.53(0.35-0.76)$ & $0.45(0.40-0.70)$ & $0.51(0.37-0.70)$ & $0.48(0.29-0.63)$ \\
\hline Grupo B & $0.51(0.40-0.72)$ & $0.58(0.48-0.96)$ & $0.58(0.41-0.77)$ & $0.47(0.37-0.92)$ \\
\hline \multicolumn{5}{|c|}{ Bilirrubina indirecta (mg/dL) } \\
\hline Grupo A & $0.30(0.21-0.52)$ & $0.30(0.20-0.49)$ & $0.30(0.20-0.50)$ & $0.30(0.19-0.45)$ \\
\hline Grupo B & $0.30(0.22-0.49)$ & $0.40(0.30-0.70)$ & $0.33(0.26-0.53)$ & $0.30(0.21-0.60)$ \\
\hline \multicolumn{5}{|c|}{ Bilirrubina directa (mg/dL) } \\
\hline Grupo A & $0.20(0.13-0.22)$ & $0.18(0.14-0.24)$ & $0.18(0.10-0.24)$ & $0.15(0.12-0.21)$ \\
\hline Grupo B & $0.19(0.14-0.27)$ & $0.21(0.18-0.30)$ & $0.19(0.14-0.22)$ & $0.18(0.12-0.28)$ \\
\hline \multicolumn{5}{|c|}{ Fosfatasa alcalina (U/L) } \\
\hline Grupo A & $80(70-95)$ & $84(68-107)$ & $82(71-98)$ & $84(74-91)$ \\
\hline Grupo B & 81 (71-92) & 77 (68-92) & 75 (64-92) & 78 (66-89) \\
\hline \multicolumn{5}{|c|}{ Glucosa en ayuno $(\mathrm{mg} / \mathrm{dL})^{*}$} \\
\hline Grupo A & $96 \pm 25$ & $91 \pm 20$ & $92 \pm 8$ & $94 \pm 6$ \\
\hline Grupo B & $90 \pm 19$ & $90 \pm 9$ & $94 \pm 15$ & $91 \pm 10$ \\
\hline \multicolumn{5}{|c|}{ Colesterol total $(\mathrm{mg} / \mathrm{dL})^{\star}$} \\
\hline Grupo A & $168 \pm 25$ & $173 \pm 32$ & $179 \pm 33$ & $169 \pm 29$ \\
\hline Grupo B & $161 \pm 24$ & $159 \pm 35$ & $157 \pm 30$ & $167 \pm 22$ \\
\hline \multicolumn{5}{|c|}{ LDL-C (mg/dL)* } \\
\hline Grupo A & $109 \pm 28$ & $112 \pm 27$ & $121 \pm 32$ & $111 \pm 30$ \\
\hline Grupo B & $100 \pm 23$ & $104 \pm 26$ & $98 \pm 35$ & $108 \pm 24$ \\
\hline \multicolumn{5}{|c|}{ HDL-C (mg/dL)* } \\
\hline Grupo A & $47 \pm 20$ & $43 \pm 10$ & $41 \pm 11$ & $50 \pm 24$ \\
\hline Grupo B & $47 \pm 18$ & $46 \pm 9$ & $56 \pm 54$ & $47 \pm 9$ \\
\hline \multicolumn{5}{|c|}{ Triglicéridos (mg/dL) } \\
\hline Grupo A & $116(90-153)$ & $133(99-183)$ & $113(88-177)$ & $115(100-159)$ \\
\hline Grupo B & $102(85-140)$ & $100(76-130)$ & 103 (92-159) & $104(86-126)$ \\
\hline
\end{tabular}

Mediana (rango intercuartílico). * Media y desviación estándar.

Valores en negrita tuvieron una $p<0.05$. El resto no fue significativo.

$\mathrm{ALT}$ = alanina aminotransferasa; $\mathrm{AST}$ = aspartato aminotransferasa; GGT = gamma-glutamil transferasa. 


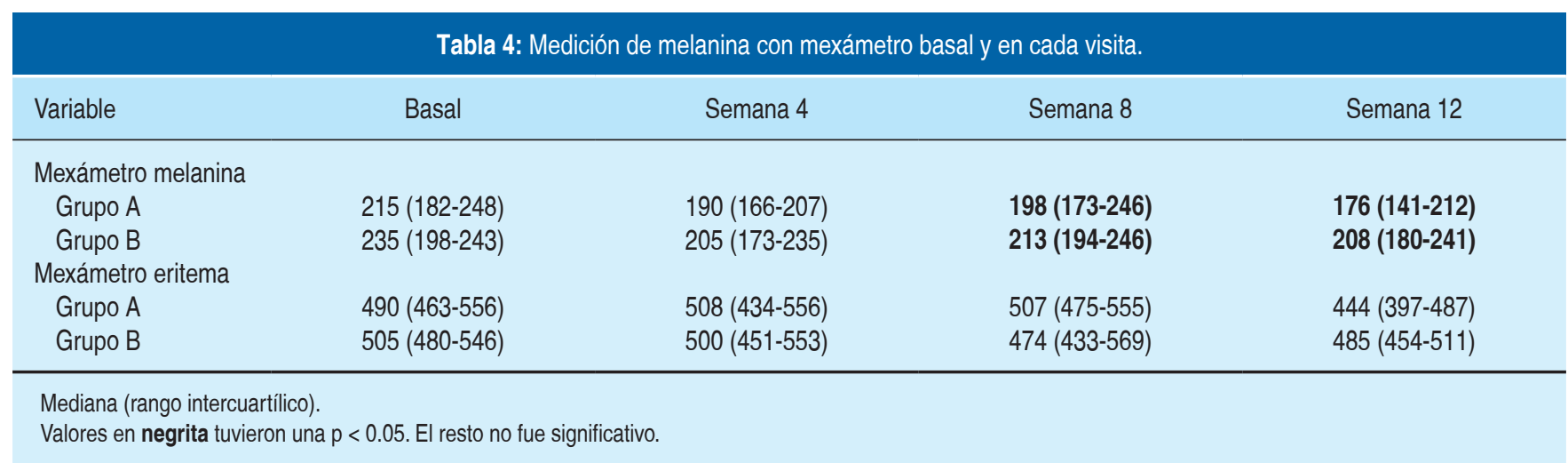

cativa entre administrar $10 \circ 20 \mathrm{mg} /$ día de isotretinoína para el tratamiento de la rosácea. En nuestro estudio, ambos esquemas terapéuticos lograron una reducción clínicamente significativa del número de pápulas y pústulas, así como del eritema. Al realizar el análisis por intención a tratar, considerando las pérdidas en ambos grupos, los resultados fueron similares. Los efectos adversos clínicos y de parámetros de laboratorio fueron similares en ambos grupos de tratamiento. La eficacia de ambos esquemas de isotretinoína se vio reflejada en la mejoría de la calidad de vida de los pacientes a las 12 semanas del tratamiento.

En la última revisión sistemática Cochrane, se concluyó que las dosis bajas de isotretinoína son de 3 a $36 \%$ más efectivas que la doxiciclina a dosis de $50-100 \mathrm{mg} /$ día para el tratamiento de la rosácea pápulo-pustular,

\begin{tabular}{|cccc}
\hline \multicolumn{4}{|c}{ Tabla 5: Efectos adversos durante cada visita. } \\
\hline & Grupo A & Grupo B & $p$ \\
\hline Efectos adversos (\%) & & & \\
Semana 4 & 52.90 & 58.80 & 0.466 \\
Semana 8 & 26.50 & 26.50 & 1.000 \\
Semana 12 & 5.90 & 5.90 & 1.000 \\
Dolor (\%) & & & \\
Semana 4 & 17.6 & 11.8 & 0.734 \\
Semana 8 & 8.8 & 2.9 & 0.307 \\
Semana 12 & 2.9 & 2.9 & 1.000 \\
Xerosis (\%) & & & \\
Semana 4 & 23.5 & 29.4 & 0.392 \\
Semana 8 & 8.8 & 14.7 & 0.355 \\
Semana 12 & 2.9 & 5.9 & 0.500 \\
Prurito (\%) & & & \\
Semana 4 & 14.7 & 8.8 & 0.355 \\
Semana 8 & 5.9 & 0.0 & 0.246 \\
Semana 12 & 2.9 & 5.9 & 0.500 \\
\hline
\end{tabular}

con un RR de 1.18 (IC95\% 1.03-1.36). ${ }^{14}$ El primer estudio que demostró la eficacia de la isotretinoína para el tratamiento de la rosácea fue realizado por Gollnick. En este estudio se observó que la isotretinoína a dosis de $0.3 \mathrm{mg} / \mathrm{kg}$ de peso es superior al placebo e igual que la doxiciclina para lograr una reducción de $90 \%$ de las lesiones inflamatorias. También evidenciaron que es más segura la dosis de $0.3 \mathrm{mg} / \mathrm{kg}$ que la de $0.5 \mathrm{mg} / \mathrm{kg}$, la cual ocasiona más cuadros de dermatitis facial como efecto secundario. ${ }^{15}$ Estos resultados fueron similares a los reportados posteriormente por Sbidian en un estudio con pacientes diagnosticados con rosácea de difícil tratamiento. En este estudio se demostró que la dosis de $0.25 \mathrm{mg} / \mathrm{kg}$ peso es efectiva para el tratamiento de la rosácea pápulo-pustular resistente a tratamiento de primera línea. ${ }^{16}$ En el nuestro, a las 12 semanas hubo una disminución promedio de $86 \%$ de pápulas y de $83 \%$ de pústulas en ambos grupos, sin encontrarse diferencias ni clínicas ni estadísticamente significativas con ambos esquemas de isotretinoína (10 mg vs $20 \mathrm{mg} /$ día). La dosis de $10 \mathrm{mg} /$ día es equivalente a $0.1 \mathrm{mg} / \mathrm{kg}$ peso al día y la de $20 \mathrm{mg} /$ día a la de $0.3 \mathrm{mg} / \mathrm{kg}$ de peso.

Los estudios previos no exploraron el porcentaje de recidiva de las lesiones inflamatorias al término del tratamiento con isotretinoína. Aunque en nuestro estudio pocos pacientes acudieron a la cita de seguimiento, a las 24 semanas se observó que 68 y $71 \%$ de los pacientes en los grupos de 10 vs 20 mg/día, presentaron recidiva de las lesiones inflamatorias. Es decir, la recidiva parece ser similar con ambos esquemas terapéuticos. Los dermatólogos debemos considerar que posterior a un esquema de 12 semanas de isotretinoína, los pacientes presentarán recidiva.

Un resultado interesante medido de manera objetiva fue la valoración de la melanina con el uso del mexámetro, con el cual se documentó que el grupo que recibió $20 \mathrm{mg} /$ día de isotretinoína presentó una reducción 
del índice de melanina a las semanas ocho y 12 , ( $p<$ 0.05). En la literatura existe un reporte de caso de una paciente que padecía tanto acné como melasma, y que al recibir isotretinoína tuvo una mejoría importante en el melasma. ${ }^{17}$ Se conocen los efectos de la tretinoína tópica en el tratamiento del melasma; sin embargo, no se conocen muy bien los efectos del tratamiento con retinoides orales en pacientes con melasma.

Es importante señalar que la principal limitación de este estudio fue el no completar el tamaño de muestra calculado para demostrar equivalencia de ambos esquemas terapéuticos (valor beta final de la muestra de 0.05). Sin embargo, las fortalezas metodológicas del mismo fueron la aleatorización de la asignación a las intervenciones, la evaluación de las lesiones inflamatorias por parte de un investigador cegado a las intervenciones y la medición objetiva del eritema y de la melanina utilizando la sonda mexámetro; así como el registro de los efectos secundarios a través de las bitácoras de los pacientes y de los estudios de laboratorio.

\section{CONCLUSIONES}

Los resultados obtenidos en este estudio sustentan que la administración de $10 \mathrm{mg} /$ día de isotretinoína para el tratamiento de la rosácea pápulo-pustular es similar a la dosis de $20 \mathrm{mg} / \mathrm{día}$. Y que aunque en nuestra muestra la incidencia de efectos secundarios fue similar, en estudios previos se ha observado que una menor dosis diaria de isotretinoína reduce la incidencia de efectos adversos graves que ameriten la suspensión del tratamiento.

\section{AGRADECIMIENTOS}

A Quetzalli Georgina Chirino-Castillo y Germán GuerreroOliva por su participación en el reclutamiento de pacientes y apoyo en los procedimientos del estudio. Las cápsulas de isotretinoína fueron donadas por el laboratorio Progela Pharma, marca Gelcupro. El personal del laboratorio Progela Pharma no estuvo involucrado en el diseño, reclutamiento ni en ningún procedimiento del estudio, incluido el análisis estadístico y la redacción del manuscrito.

\section{REFERENCIAS}

1. Van Zuuren EJ. Rosacea. New Engl J Med. 2017;377:1754-1764. Available in: https://doi.org/10.1056/NEJMcp1506630.

2. Van Zuuren EJ, Fedorowicz Z, Carter B, Van der Linden MM, Charland L. Interventions for rosacea. Cochrane Database Syst Rev. 2015;2015:CD003262.

3. Powell FC. Rosacea. N Engl J Med. 2005;352:793-803.
4. Gether L, Overgaard LK, Egeberg A, Thyssen JP. Incidence and prevalence of rosacea: a systematic review and meta-analysis. $\mathrm{Br} J$ Dermatol. 2018;179:282-289. Available in: https://doi.org/10.1111/bjd.16481.

5. Culp B, Scheinfeld N. Rosacea: a review. P T. 2009;34:38-45.

6. Amir A, Scott AD, Amy JM, Steven F. Rosacea in skin of color: not a rare diagnosis. Dermatol Online J. 2014;20(10):13030/qt1mv9r0ss.

7. Marson JW, Baldwin HE. Rosacea: a wholistic review and update from pathogenesis to diagnosis and therapy. Int $J$ Dermatol. 2020;59:e175-e182. Available in: https://doi.org/10.1111/ijd.14757.

8. Gallo RL, Granstein RD, Kang S, Mannis M, Steinhoff M, Tan J et al. Standard classification and pathophysiology of rosacea: The 2017 update by the National Rosacea Society Expert Committee. J Am Acad Dermatol. 2018;78:148-155. Available in: https://doi. org/10.1016/j.jaad.2017.08.037.

9. Zeichner JA, Eichenfield LF, Feldman SR, Kasteler JS, Ferrusi IL. Quality of life in individuals with erythematotelangiectatic and papulopustular rosacea: findings from a web-based survey. J Clin Aesthet Dermatol. 2018;11:47-52.

10. Thiboutot D, Anderson R, Cook-Bolden F, Draelos Z, Gallo RL, Granstein RD et al. Standard management options for rosacea: the 2019 update by the National Rosacea Society Expert Committee. J Am Acad Dermatol. 2020;82:1501-1510. Available in: https://doi. org/10.1016/j.jaad.2020.01.077.

11. Hoting E, Paul E, Plewig G. Treatment of rosacea with isotretinoin. Int J Dermatol. 1986;25:660-663. Available in: https://doi. org/10.1111/j.1365-4362.1986.tb04533.x.

12. Rademaker M. Very low-dose isotretinoin in mild to moderate papulopustular rosacea; a retrospective review of 52 patients. Australas J Dermatol. 2018;59:26-30. Available in: Available in: https:// doi.org/10.1111/ajd.12522.

13. Sbidian E, Vicaut E, Chidiack H, Anselin E, Cribier B, Dréno B et al. A randomized-controlled trial of oral low-dose isotretinoin for difficult-totreat papulopustular rosacea. J Invest Dermatol. 2016;136:1124-1129. Available in: https://doi.org/10.1016/j.jid.2016.01.025.

14. Van Zuuren EJ, Ben Carter ZF, van der Linden M, Charland L. Interventions for rosacea. Cochrane Database Syst Rev. 2015;2015:CD003262.

15. Gollnick H, Blume-Peytavi U, Szabó EL, Meyer KG, Hauptmann P, Popp $G$ et al. Systemic isotretinoin in the treatment of rosacea -doxycyclineand placebo-controlled, randomized clinical study. J Dtsch Dermatol Ges. 2010;8:505-515. doi: 10.1111/j.1610-0387.2010.07345.x.

16. Sbidian E, Vicaut É, Chidiack H, Anselin E, Cribier B, Dréno B, Chosidow $O$. A randomized-controlled trial of oral low-dose isotretinoin for difficult-to-treat papulopustular rosacea. J Invest Dermatol. 2016;136:1124-1129. doi: 10.1016/j.jid.2016.01.025.

17. Lenz CR, Aldrich SL. Effect of systemic isotretinoin treatment on melasma: a case study and literature review. Clinical Dermatology Open Access Journal.2019; 1-6. Available in: https://doi.org/10.23880/cdoaj-16000182.

Correspondencia:

Martha Alejandra Morales-Sánchez

José María Vértiz Núm. 464,

Col. Buenos Aires, 06840,

Alcaldía Cuauhtémoc, Ciudad de México, México, E-mail: marthamoralessanchez@gmail.com. 